\title{
MEASURING AND IMPROVING CHILDREN'S READING ALOUD ATTRIBUTES BY COMPUTERS
}

\author{
MAREK NAGY \\ Faculty of Mathematics, Physics and Informatics, \\ Comenius University in Bratislava, Slovakia
}

\begin{abstract}
NAGY, Marek: Measuring and Improving Children's Reading Aloud Attributes by Computers. Journal of Linguistics, 2017, Vol. 68, No 2, pp. 278 - 286.
\end{abstract}

\begin{abstract}
In this paper, method of an automated measuring reading aloud attributes is presented. The forced alignment as a part of speech recognition technique is used. The recorded reading aloud is forced aligned to the known text and the attributes are computed from it. The tempo and fluency of children are monitored and used for an individual motivation. The length of the read text is chosen according to readers' skills so that children end up reading at about the same time and poor readers are not frustrated. This approach has been tested and improved at the elementary school for five years and brought positive results.

Keywords: speech recognition, teaching reading, reading aloud
\end{abstract}

\section{INTRODUCTION}

The reading is the main skill that children acquire in elementary schools. Step by step, children learn to recognize letters and combine them into words. When teachers want an effective feedback the children must read aloud. The teacher listens to the reading and corrects errors. Computer applications that use speech recognition can also do it automatically [1], [2], [3]. The children improve their reading aloud and read faster and smoother. In the second grade, children read longer texts that develop their reading skills. Reading aloud is only a temporary level to silent reading. However, it will be better to remain at this level some time because children have to learn to read words as a whole with its meaning at first. Whether it happens it is possible to find out by watching the tempo and fluency of reading. If children read fast it is clear that they are not syllabifying words. This means that they know and understand the words. It can be supposed that if the tempo and fluency of reading aloud is higher then children understand more words of the text. Monitoring of the reading tempo and fluency is an important thing for teachers. To help teachers these two attributes will be automatically measured by computer.

\section{READING ALOUD ATTRIBUTES}

The progress in the reading aloud can be measured by the tempo of reading. It is a common attribute that has been being used for long time. To measure this attribute a stopwatch is only needed. Teachers prepare text, which has already countered and signed words. Children start reading when the teacher starts the stopwatch. After one minute the teacher stops the stopwatch, counts the correctly read words and computes 
words correct per minute (WCPM) ratio [4]. This procedure can be simply used in a computer. Children start reading into a microphone and when they finish the computer stops recording. The whole reading time is computed from the length of the recorded speech. A problem is at the beginning and end of the recording where silence can occur. It can be reduced by a silence detector. But a main problem is with automatic checking correctly read words. A computer speech recognition is used to identify words in recordings [1], [2], [5]. The methods are based on Hidden Markov Models (HMM) recognition approach. HMMs are trained from speech data as subunits e.g. phones, triphones, ... [6] A substantial part of the recognizer is a recognition grammar or network, which determines possible sequences of subunits that can be recognized. To design such grammar is quite a problem. If the grammar is too complex (i.e. many possible sequences) an accuracy of the recognizer is lower and a processor time consumption is higher. In case of children's reading it must also be taken into account a fact that children can read aloud meaningless words e.g. when they syllabify words or they omit a phone.

Another requirement is a real-time response. Children will see their reading attributes immediately after they have read the text. Our experiments showed that if the feedback is late it is not a motivation factor.

We were used our Slovak speech recognizer, that was trained on children's speech data from Multimedia Reader [7]. The chosen HMM subunits are triphones. The isolated word accuracy is $98 \%$ with small dictionary (ca. hundred words) and phone accuracy is $88 \%$. The recognizer is running on a server and must manage ca 20 reading children at once. If children are reading the same text then the recognition network can be shared what reduces a memory consumption.

We start to design grammar from the forced alignment approach. It means that if we know a potential transcription (the text), then we can compute time boundaries of individual words (of the text). Ideally, the transcription is a plain text as can children see and read from the computer monitor (see Figure 1). The syntax is taken from [6]. \#sil represents a special unit that models silence. It can absorb a short or long pause between words.

$$
\text { \#sil word \#sil word, \#sil word \#sil ... }
$$

Fig. 1. The optimistic transcription-grammar used for the forced alignment

However, when children make mistakes the transcription has to contain an appropriate correction. The typical mistake is repeating words (a double/triple reading) or omitting words (due to inattention). A situation of swapping words is infrequent and can be modeled as an incorrect reading and omitting. Therefore, the transcription is made as a recognition network that is build from a recognition grammar (see Figure 2). The curly brackets mean that their content can be repeating zero or more times.

$$
\text { \#sil }\left\{\text { word }_{1} \# \operatorname{sil}\right\} \quad\left\{\text { word }_{2} \# \operatorname{sil}\right\} \quad\left\{\operatorname{word}_{3} \# \operatorname{sil}\right\} \text { \#sil ... }
$$

Fig. 2. The repeating and omitting transcription-grammar used for the forced alignment. The curly brackets denote zero or more repetitions. 
This approach consumes too much processor time to compute the best sequence of words because the number of subsequences (with repetitions and omissions) is very high for long texts (more than 200 words). In this case, unfortunately, the recognizer will accept the reading with many omitted words, too. E.g. the tempo will be computed from one word only, what is undesirable. Therefore, the grammar was changed (see Figure 3). The angle brackets mean one or more times repeating. Now, words can't be omitted. If children omit too many words then the recognizer will not find out a result and the whole reading is rejected. That is the reason why we introduce possibility for the recognizer to reject the reading. Our goal is that children will read the whole text correctly and if it needed they can read it again. It is an advantage against approaches where the recordings are static and processed off-line. Our experiments showed that the omitting is not a significant problem and therefore the rejecting can appears only infrequently.

$$
\# \text { sil < } \operatorname{word}_{1} \# \mathrm{sil}><\operatorname{word}_{2} \# \mathrm{sil}><\operatorname{word}_{3} \# \mathrm{sil}>\ldots
$$

Fig. 3. The repeating and omitting transcription-grammar used for the forced alignment. The angle brackets denote one or more repetitions.

According to [6] the recognition network is constructed using a dictionary that contains phonetic sequences for words. One word can have more phonetic sequences. See Figure 4.

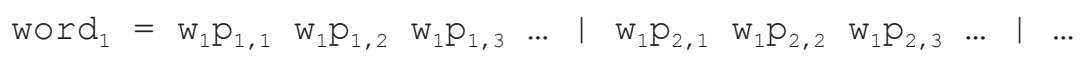

Fig. 4. The different phonetic sequences of the word $_{1}$. The vertical bars denote alternatives.

If we want to omit dictionary then the phonetic sequences have to be written into the grammar directly. See Figure 5. Of course, the dictionary must exist but it will contain direct phone-phone mapping only.

$$
\begin{aligned}
& \text { \#w0 } \\
& <\left(\begin{array}{lllllllll}
\mathrm{w}_{1} \mathrm{P}_{1,1} & \mathrm{w}_{1} \mathrm{P}_{1,2} & \mathrm{w}_{1} \mathrm{P}_{1,3} & \ldots & \mid & \mathrm{w}_{1} \mathrm{p}_{2,1} & \mathrm{w}_{1} \mathrm{P}_{2,2} & \mathrm{w}_{1} \mathrm{p}_{2,3} & \ldots
\end{array}\right) \quad \# \mathrm{w} 1> \\
& <\left(\begin{array}{llllllll}
\mathrm{w}_{2} \mathrm{P}_{1,1} & \mathrm{w}_{2} \mathrm{P}_{1,2} & \mathrm{w}_{2} \mathrm{P}_{1,3} & \ldots & \mid \mathrm{w}_{2} \mathrm{P}_{2,1} & \mathrm{w}_{2} \mathrm{P}_{2,2} & \mathrm{w}_{2} \mathrm{P}_{2,3} & \ldots
\end{array}\right) \quad \# w 2> \\
& <\left(\begin{array}{lllllllll}
\mathrm{w}_{\mathrm{N}} \mathrm{p}_{1,1} & \mathrm{w}_{\mathrm{N}} \mathrm{p}_{1,2} & \mathrm{w}_{\mathrm{N}} \mathrm{p}_{1,3} & \ldots & \mid & \mathrm{w}_{\mathrm{N}} \mathrm{p}_{2,1} & \mathrm{w}_{\mathrm{N}} \mathrm{P}_{2,2} & \mathrm{w}_{\mathrm{N}} \mathrm{p}_{2,3} & \ldots
\end{array}\right) \quad \# \mathrm{wN}>
\end{aligned}
$$

Fig. 5. The grammar used for the forced alignment. Every word is substituted by its phonetic transcriptions. Special non-terminals \#w0- \#wN (equal to \#sil) are introduced.

The terminals word ${ }_{i}$, which represent composed HMMs, can't be used because phones are the terminals (HMMs) now. Therefore, we are using non-terminals \#w1$\#$ wN that represents silence (equal to \#sil) between two neighboring words. The \#w0 is a special case. The recognition algorithm (Vitterbi search) will generate sequence of phones, which is intermittented by silences $\# \mathrm{w} 0-\# \mathrm{wN}$. The time boundaries of words can be deduced from the boundaries of silences \#w0-\#wN. 


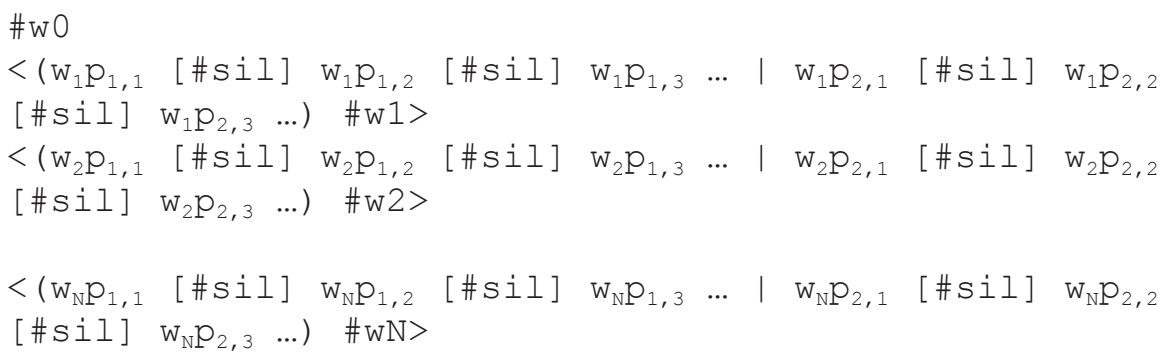

Fig. 6. The grammar used for the forced alignment. Every word is substituted by its phonetic transcriptions. Special non-terminals \#w0-\#wN (equal to \#sil) are introduced and \#sil is inserted between phones.

When children are reading they can syllabify words (or can use an unwanted double/triple reading). It introduces inaccuracy in a time alignment. So silences are inserted between phones, too (see Figure 6). Now, the algorithm is able to compute the time boundaries of words relatively good. The side effect is determination of silence between phones. It helps to express the fluency.

The grammar from Figure 6 is based on the assumption that children have read the whole text. If they leave out (or swap) more words (grater then ca. 10) then Vitterbi alignment algorithm [6] can't find the appropriate path - the word sequence. In our case it is not a problem because children are motivated by this way to read the whole text without too many mistakes. However, if the problem occurs children will read the text again.

Now we can compute time of the reading (in seconds), summary time of the words and summary time of the silences. It must be met. If we have a number of words the tempo can be computed as

$$
\text { tempo }_{W O}=\frac{N_{W O}}{T} 60 \quad[\text { words } / \text { min }
$$

The tempo based on a number of words is not very reliable. Our experiments showed that the tempo based on a number of syllables is better, because our texts are not normalized and it happens that the text contains too many -3 and more - syllabic words. (The tempo in the prosody is also based on syllables.) The syllables can be easily counted as a number of vowels. Diphthongs are a special case. And syllabic consonants $\mathrm{r}, 1$ surrounded by consonants are understood as syllables.

$$
\text { tempo }_{S Y}=\frac{N_{S Y}}{T} 60 \quad[\text { syllables } / \mathrm{min} \text {. }
$$

We proposed computing the fluency as:

$$
\text { fluency }=\frac{T_{W}}{T_{S}}
$$

The tempo and fluency are computed for the text as a whole and have global meaning. Our experiments showed that the attributes change during reading. In the future, the graph of it can help teacher identify the difficult sequences of words. 


\section{MEASURING THE READING ATTRIBUTES ON ELEMENTARY SCHOOL}

Children read texts at school at lessons. A special subject has been introduced for this reason called Multimediálne čítanie, 'Multimedia Reading'. It is done once in a week. Children use computers with the web application Multimediálna čítanka, 'Multimedia Reader' [7] that records their reading aloud by a microphone. When they finish the computer shows a simple one line diagram of the tempo and the fluency where children see their progress. Children are rewarded when they go up. It is an individual approach because a child only compares his/her actual personal performance with his/her previous ones. Children immediately see whether their attributes are rising or not. If they are not satisfied they can read again and improve their rating. After the corrective reading children can mostly see an instant progress, which is a motivating factor. The second and the other attempts make sense only for instant evaluation on the actual lesson. On the next lesson, the first attempts of previous lessons are only used in all statistics and graphs.

In this manner, children proceeded from the $1^{\text {st }}$ to the $3^{\text {rd }}$ grade. It happens that in $3^{\text {rd }}$ grade the proficient readers tend to read aloud too quickly, so that they are not understood by others. E.g. they swallow word endings. To suppress inarticulate readings we set the limit on the tempo as $\mathbf{2 6 0}$ syllables $/ \mathrm{min}$. It is ca. 130 words $/ \mathrm{min}$. Kids who exceeded the limit were rewarded regardless whether they go up or down. Similarly, the fluency has limit set to 3 . We determined these limits experimentally. We started at 300 syllables/min but many children were frustrated by impossibility to reach it. Teachers also expressed objections that this limit is too high. We think that it relates to physical parameters of a vocal tract.

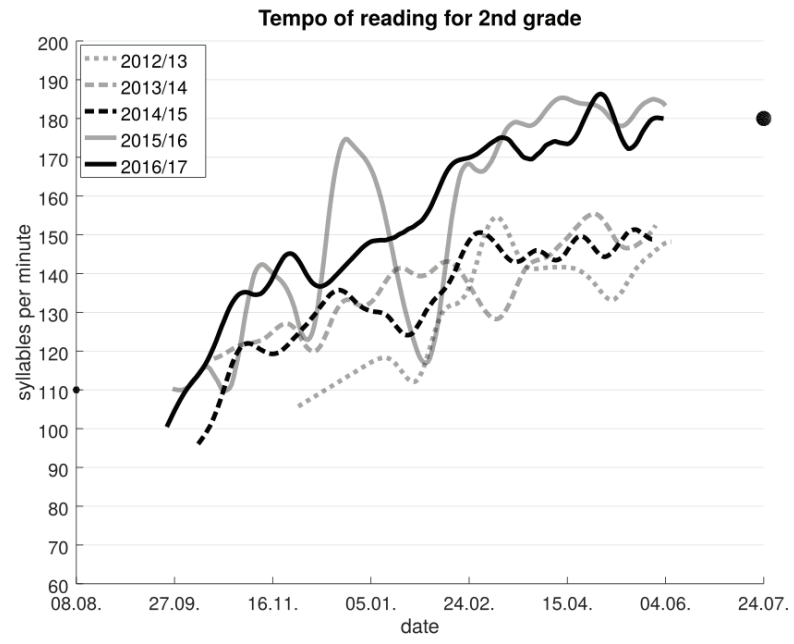

Fig. 7a. The graph of the average tempo of reading-aloud for $2^{\text {nd }}$ graders. The actual 2016-17 and previous school years are showed. 


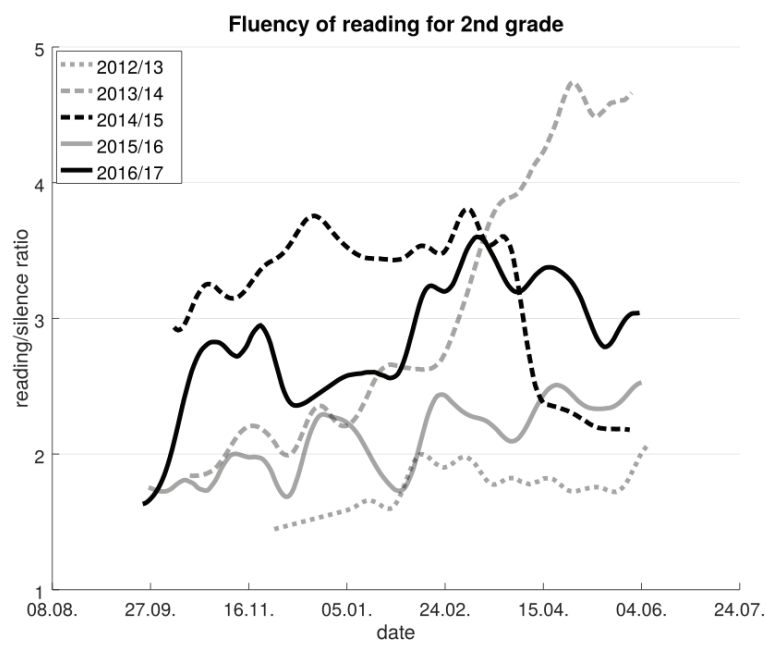

Fig. 7b. The graph of the average fluency of reading-aloud for $2^{\text {nd }}$ graders. The actual 2016-2017 and previous school years are showed.

On the Figures $7 \mathrm{a}$ and $7 \mathrm{~b}$, improvements of the tempo and fluency are shown. Five consecutive school years are included. It were 2 nd grade children in age $7-8$ yo. The graph of averages is constructed on week base and every child performance is included only once a week. The yellow circles on boundaries of the graph (Figure 7a) correspond to average oral reading fluency norms, which are taken from [4]. As can be seen, within five years, we managed to increase the tempo of reading at an adequate level. The school years 2015/16 and 2016/17 are already within the norm. It should be noted that the number of syllables has been extrapolated from the number of words in the norm. It is ca. twice the number. We make average ratio for all 2069 usable texts included in the Multimedia reader [7] and it gives syllables/words ratio equal to 1.9. The number of children, which are taken, is presented in Table 1.

\begin{tabular}{|l|r|r|r|}
\hline school year & girls & boys & total \\
\hline $2012 / 13$ & 21 & 16 & 37 \\
\hline $2013 / 14$ & 24 & 15 & 39 \\
\hline $2014 / 15$ & 21 & 19 & 40 \\
\hline $2015 / 16$ & 12 & 22 & 34 \\
\hline $2016 / 17$ & 29 & 19 & 48 \\
\hline
\end{tabular}

Tab. 1. The number of $2^{\text {nd }}$ grade children in school years

Statistical graphs for teachers and parents are more detailed as it can be seen on Figure 8. There are two examples. On the left side is a worse (below average) reader and on the right a better (above average) who is attacking the limit 260 syllables/ $\mathrm{min}$. The graphs show all improvement of reading from the $1^{\text {st }}$ to $4^{\text {th }}$ grade in general. The teacher can compare the child with the mean of all class or with the mean of all grade. It gives teachers a better chance to synchronize performances of kids. 

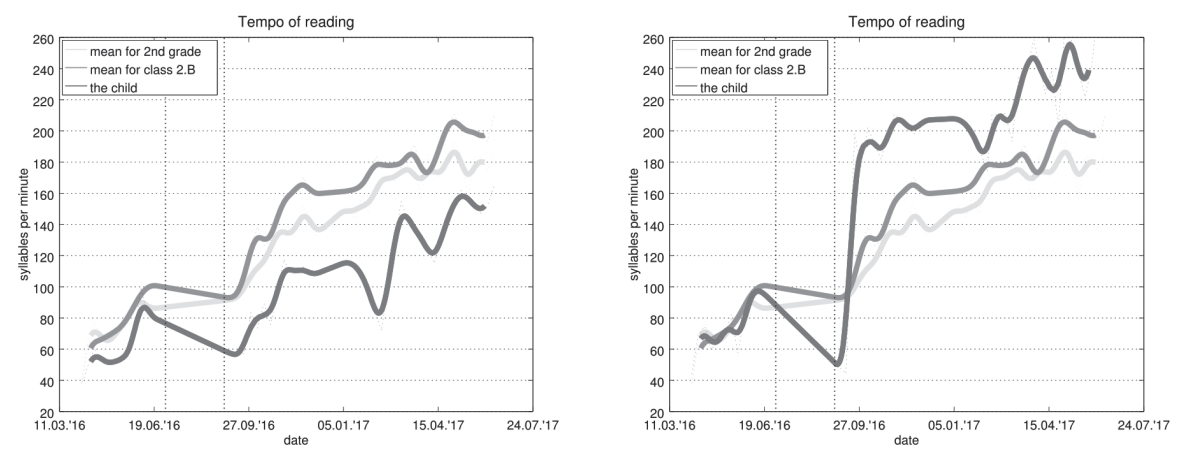

Fig. 8. The graph of the reading tempo of two 2 nd graders. The graph shows the improving of reading skills from the 1 st grade to the 2 nd grade. The middle strip bounds summer holidays.

\section{CHOOSING APPROPRIATE TEXT LENGTH FOR READING}

At the beginning the length of the text that children are reading-aloud was determined roughly. On the $1^{\text {st }}$ grade shorter and on the $2^{\text {nd }}$ grade longer. But a problem appears. The children did not end at same time in reading. Better readers read the text sooner and started to have a fun. From the beginning, some extra activities were provided, but then kids were dispersed and could not concentrate on continuing the work with the text. How fast the text is read, depends on the tempo.

The development of the mean of tempo can be analyzed from experimental data. Typical standard deviation of the tempo was experimentally measured as from our collected data. If we imagine Gaussian distribution then the vast majority of readers will have the reading tempo in interval . Concretely, statistically, $68 \%$ of children. Now we can compute what time difference will be between a worse and a better reader. We must choose the appropriate tempo in syllables per minute. If we denote number of syllables of text by we can write:

$$
\begin{gathered}
\Delta t=\frac{N_{S Y}}{\mu-\sigma}-\frac{N_{S Y}}{\mu+\sigma} \quad[\mathrm{min}] \\
\Delta t=N_{S Y} \frac{2 \sigma}{\mu^{2}-\sigma^{2}}
\end{gathered}
$$

It is good if children finish reading aloud at about the same time. If we choose a time difference, then we can compute how many syllables the text must have. See formula 6 .

$$
N=\Delta t \frac{\mu^{2}-\sigma^{2}}{2 \sigma} \quad[\text { syllables }
$$




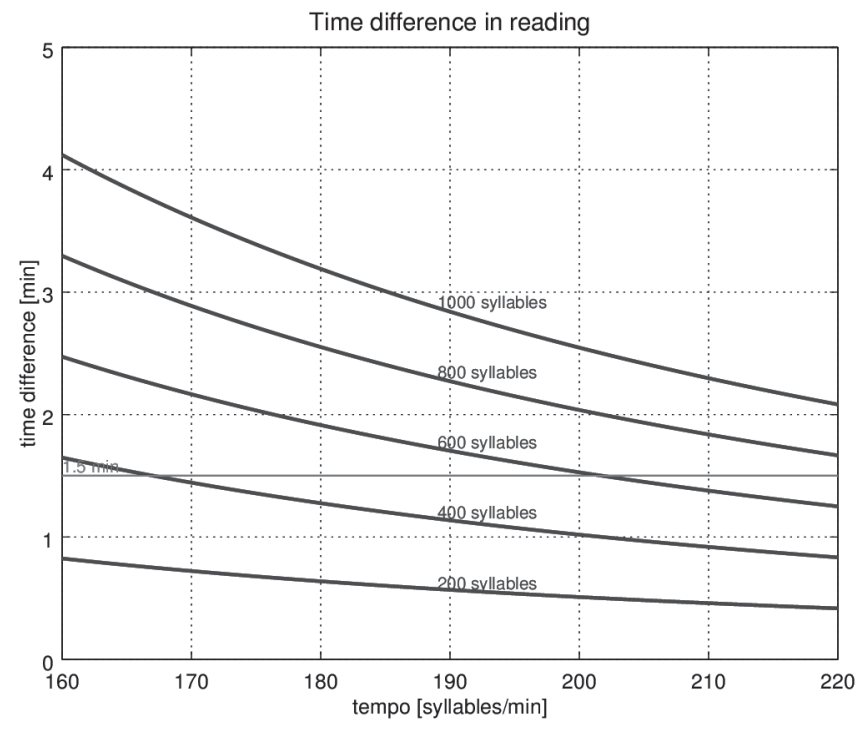

Fig. 9a. Graphs of dependency among the tempo and the finish time difference

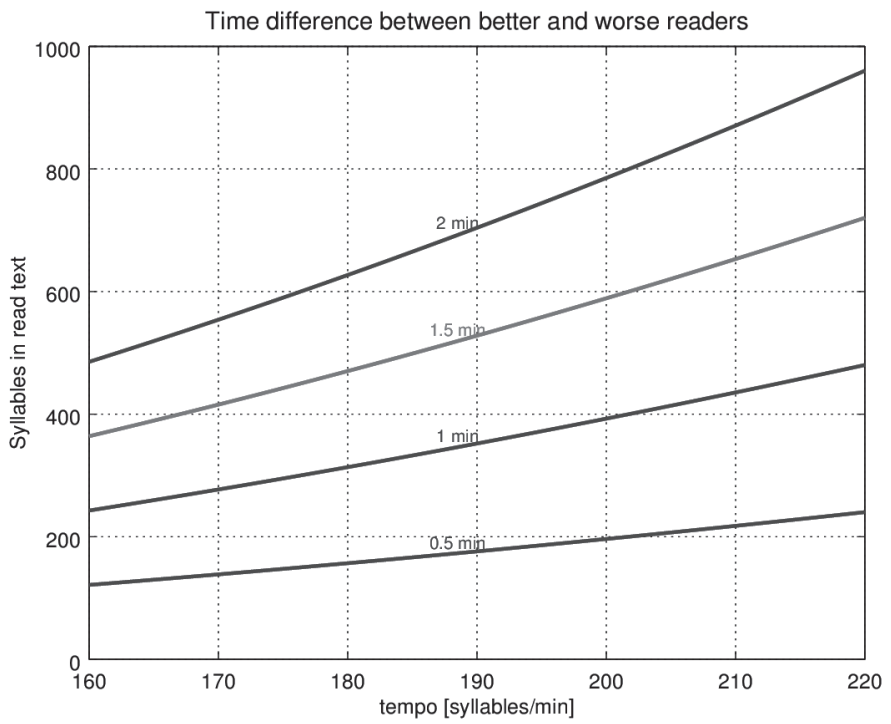

Fig. 9b. Graphs of dependency among the tempo and the text length

We have experimentally found out that the time difference must be less than 1.5 minutes. On the Figures $9 \mathrm{a}$ and $9 \mathrm{~b}$ are graphs for the tempo $160-220$ syllables/ $\mathrm{min}$. According to the Figure $7 \mathrm{a}$ the $2^{\text {nd }}$ graders read ca. 180 syllables/min at the end of school year. The suitable text should have length ca. 500 syllables (ca. 250 words). 


\section{CONCLUSION}

The measuring reading attributes, which is presented above, has brought automation in monitoring reading skills of children. Among other things, automation also brings a certain degree of objectivity. Teachers can compare children's performances and identify problematic ones. On the other hand, kids are proceeding in individual manner and are motivated to overcome themselves. Children are rewarded relative to their own performance and not on established values. This is particularly important for poor readers, who would be everytime at the end. The mentioned approach has improved the overall readership levels as is shown in Figure 7. Another motivating factor is the time aspect. It is important that children end up reading a text at about the same time. Otherwise, they are frustrated because they see that they are objectively slower. To avoid this problem it is necessary to choose an appropriate range of texts. If children end up reading at about the same time, it allows us to better manage the teaching, so children have no time for distraction.

\section{References}

[1] Mostow, J. and Roth, S. F. (1995). Demonstration of a reading coach that listens. In Hauptmann, A. G., editor, Proceedings of the 8th Annual ACM Symposium on User Interface and Software Technology. UIST '95, pages 77-78, ACM, New York, NY.

[2] Bolaños, D., Cole, R. A., Ward, W., Borts, E., and Svirsky, E. (2011). Flora: Fluent oral reading assessment of children's speech. ACM Trans. Speech Lang. Process., 7(4):16:1-16:19.

[3] Patel, R. and Furr, W. (2011). Readn'karaoke: Visualizing prosody in children's books for expressive oral reading. In Proceedings of the SIGCHI Conference on Human Factors in Computing Systems. CHI '11, pages 3203-3206, ACM, New York, NY.

[4] Hasbrouck, J. and Tindal, G. A. (2006) Oral Reading Fluency Norms: A Valuable Assessment Tool for Reading Teachers. Journal of the Reading Teacher, 59:636-644.

[5] Zechner, K., Sabatini, J., and Chen, L. (2009). Automatic Scoring of Children's Read-Aloud Text Passages and Word Lists. In Proceedings of the NAACL HLT Workshop on Innovative Use of NLP for Building Educational Applications, pages 10-18, Boulder, Colorado.

[6] Young, S., Evermann, G., Gales, M., Hain, T., Kershaw, D., Liu, X., Moore, G., Odell, J., Ollason, D., Povey, D., Valtchev, V., and Woodland, P. (2006). The HTK Book Version 3.4, Cambridge University, Cambridge.

[7] Nagy, M. (2005). Multimedia Reader (Multimediálna čítanka), a web application, https:// www.mmcitanka.sk, (in Slovak). 